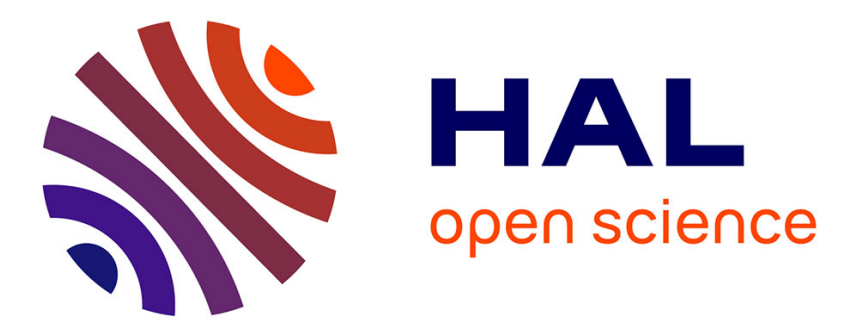

\title{
User evaluation of a haptic-enabled shared-control approach for robotic telemanipulation
}

Firas Abi-Farraj, Claudio Pacchierotti, Paolo Robuffo Giordano

\section{To cite this version:}

Firas Abi-Farraj, Claudio Pacchierotti, Paolo Robuffo Giordano. User evaluation of a haptic-enabled shared-control approach for robotic telemanipulation. IROS 2018 - IEEE/RSJ International Conference on Intelligent Robots and Systems, Oct 2018, Madrid, Spain. pp.1-8. hal-01851710

\author{
HAL Id: hal-01851710 \\ https://hal.inria.fr/hal-01851710
}

Submitted on $30 \mathrm{Jul} 2018$

HAL is a multi-disciplinary open access archive for the deposit and dissemination of scientific research documents, whether they are published or not. The documents may come from teaching and research institutions in France or abroad, or from public or private research centers.
L'archive ouverte pluridisciplinaire HAL, est destinée au dépôt et à la diffusion de documents scientifiques de niveau recherche, publiés ou non, émanant des établissements d'enseignement et de recherche français ou étrangers, des laboratoires publics ou privés. 


\title{
User Evaluation of a Haptic-enabled Shared-Control Approach for Robotic Telemanipulation
}

\author{
Firas Abi-Farraj, Claudio Pacchierotti and Paolo Robuffo Giordano
}

\begin{abstract}
Robotic telemanipulators are already widely used in nuclear decommissioning sites for handling radioactive waste. However, currently employed systems are still extremely primitive, making the handling of these materials prohibitively slow and ineffective. As the estimated cost for the decommissioning and clean-up of nuclear sites keeps rising, it is clear that one would need faster and more effective approaches. Towards this goal, in this paper we present the user evaluation of a recently proposed haptic-enabled shared-control architecture for telemanipulation. An autonomous algorithm regulates a subset of the slave manipulator degrees of freedom (DoF) in order to help the human operator in grasping an object of interest. The human operator can then steer the manipulator along the remaining null-space directions with respect to the main task by acting on a grounded haptic interface. The haptic cues provided to the operator are designed in order to inform about the feasibility of the user's commands with respect to possible constraints of the robotic system. In this paper we compared this shared-control architecture against a classical 6-DOF teleoperation approach in a real scenario by running experiments with 10 subjects. The results clearly show that the proposed shared-control approach is a viable and effective solution for improving currently-available teleoperation systems in remote telemanipulation tasks.
\end{abstract}

\section{INTRODUCTION}

Remote telemanipulation has shown significant advancements over the last years and promising results have been achieved in several robotic tasks, such as space exploration [1], minimally invasive surgery [2], sort and segregation of nuclear waste [3]-[5], telemaintenance [6], and micromanipulation [7], [8]. As teleoperation systems become more sophisticated and flexible, the environments and applications where they can be employed become less structured and predictable. In this respect, robotic telemanipulation in hazardous environments is attracting increasing attention for its potential in handling dangerous materials [3], [9] and disaster response [10][12]. Indeed, being able to remotely, intuitively, and easily manipulate objects in such scenarios could significantly extend the application range of robotic teleoperation systems. For example, Jurmain et al. [11] designed a remotely operated robot for bio-event disaster response, called HazBot. It is composed of a mobile robot equipped with a 6-degrees-offreedom (6-DoF) manipulator. The manipulator incorporates a parallel jaw gripper with a 60 pound squeeze force and a gas detector to aid in material identification. Murphy et al. [12] evaluated the use of three Unmanned Marine Vehicles (UMV) in two areas damaged by the tsunami

The authors are with the CNRS at Irisa and Inria Rennes Bretagne Atlantique, Campus de Beaulieu, 35042 Rennes Cedex, France. e-mails: \{firas.abi-farraj $\}\{$ claudio.pacchierotti $\}\{$ prg $\} @$ irisa.fr following the 2011 Tohoku Earthquake. The employed UMVs had a multi-beam imaging sonar, video, and a gripper. Stramigioli et al. [10] presented a framework for hapticenabled teleoperation of aerial robotic vehicles. The envisaged applications are inspection of piping and cabling in factories and mines, as they are often mounted inaccessibly on walls and ceilings. More recently, Abi-Farraj et al. [3] proposed a shared-control architecture for remote manipulation of nuclear waste. A visual-based autonomous algorithm regulates a subset of the gripper's degrees of freedom (DoF) in order to ease the approach towards the object of interest. The operator, on the other hand, is allowed to control the remaining nullspace motions while receiving a force feedback informative of any constraint of interest of the robotic system (e.g., joint limits, collision avoidance, and so on).

The nuclear industry is, indeed, one of the most exciting fields of applications for robotic telemanipulation; and the management of nuclear waste is a prominent issue for several countries in the world [13], [14]. Sort and segregation of the stored nuclear waste is a first step needed for the decommissioning of the site. However, current robotic systems designed for such a task provide teleoperation capabilities through extremely primitive master consoles (e.g., passive joystick or teach pendants), making the task prohibitively slow for processing large amounts of material in a reasonable time. This need motivated the European H2020 "Robotic Manipulation for Nuclear Sort and Segregation" (RoMaNS) project whose aim is to improve the currently-available teleoperation systems in nuclear environments.

When addressing remote telemanipulation tasks, the interface provided to the human operator is one of the most important components and its design has historically followed two different philosophies. A first possibility is to provide a high-fidelity haptic feedback reflecting the actual physical contacts between manipulators and environment (with all the associated stability issues in case of non-idealities, communication delays, and so on [15], [16]). A second possibility is to rely on shared-control architectures that can assist the operator in manipulation tasks by conveying 'artificial' forces cues that result from more abstract requirements/constraints (e.g., following predefined paths possibly taught by expert operators, avoiding unfeasible robot configurations, avoiding self- or obstacle-collisions) [7], [17]. The approach tested in this paper belongs to the latter category, where the focus is on achieving an efficient and fast task execution while minimizing the workload on the human operator. Therefore, the haptic interface, rather than reflecting the contact interactions of the 
slave, aims at guiding the operator to keep him away from undesired configurations of the robotic system.

As a step towards advancing the state-of-the-art in this field, we present here the evaluation of a haptic-enabled sharedcontrol architecture for telemanipulation. In our setup, an autonomous algorithm is in charge of regulating a subset of the manipulator DoF to help the human operator in approaching an object to be grasped. At the same time, the human operator steers the robotic end-effector along the remaining null-space directions with respect to the main task through a grounded haptic interface. Moreover, haptic feedback provides the human operator with information about the feasibility of the user's commands with respect to possible constraints of the robotic system, e.g., joint limits.

This shared-control algorithmic design has been preliminary presented by Abi-Farraj et al. in [3], but without providing any (even minor) user evaluation. With respect to [3], the goal of this paper is to then clearly assess the potential benefits (or cons) of a shared control architecture in remotely approaching an object to be grasped. Indeed, we note that while most of the literature concentrates on the manipulative task itself (e.g., assisted surgeries [18], or peg in hole [19]), the grasping and (in particular) the preceding approach-to-grasp phases are usually overlooked. On the other hand, the sole act of steering a remote manipulator towards a desired grasping pose (that may have been decided by the user) can be a time consuming and complex task for an operator controlling directly the 6-DoF pose of the manipulator (as in most classical setups). This is due to $(i)$ the complexity of regulating at the same time both the position and the orientation and $(\mathrm{ii})$ the typical presence of several constraints (e.g., joint limits, singularities) that further limit the operator's maneuvering dexterity (but of which the operator has no direct feeling). Therefore, the goal of this paper is to address this issue by assessing what is the best shared-control architecture for obtaining an easier, faster, and more effective robotic teleoperation for approaching an object of interest to be grasped.

The rest of the paper is organized as follows: Sect. II reviews the main algorithmic steps of the shared control architecture presented in [3], and introduces a velocityvelocity coupling as a new variation. Section III then describes the results of an experimental campaign aimed at comparing the two versions of the shared control [3] (position-velocity or velocity-velocity) against a classical full 6-DoF teleoperation. Finally, Sect. IV provides an extensive discussion of the obtained results, and draws some possible future perspectives.

\section{SHARED CONTROL ARCHITECTURE}

This Section briefly summarizes the shared-control architecture presented in [3], and discusses how it has been adapted and extended for running the intended user evaluation.

\section{A. Problem statement}

The setup we considered consists of a 6-DoF serial manipulator equipped with a gripper. The task is the remote grasping of an object. The manipulator is controlled by an operator acting on a master device and interacting with the system through haptic and visual feedback. As shown in Fig. 1, three frames of reference are considered, $\mathcal{F}_{O}$ : $\left\{\boldsymbol{O}_{O} ; \boldsymbol{X}_{O}, \boldsymbol{Y}_{O}, \boldsymbol{Z}_{O}\right\}$ attached to the object to be grasped, $\mathcal{F}_{G}:\left\{\boldsymbol{O}_{G} ; \boldsymbol{X}_{G}, \boldsymbol{Y}_{G}, \boldsymbol{Z}_{G}\right\}$ attached to the gripper and $\mathcal{F}_{B}:\left\{\boldsymbol{O}_{B} ; \boldsymbol{X}_{B}, \boldsymbol{Y}_{B}, \boldsymbol{Z}_{B}\right\}$ attached to the base of the robot. For the grasp to be successful, we assume that $Z_{G}$ should be aligned with the gripper approaching direction. This geometric constraint is actively enforced by the autonomous part of the shared-control algorithm.

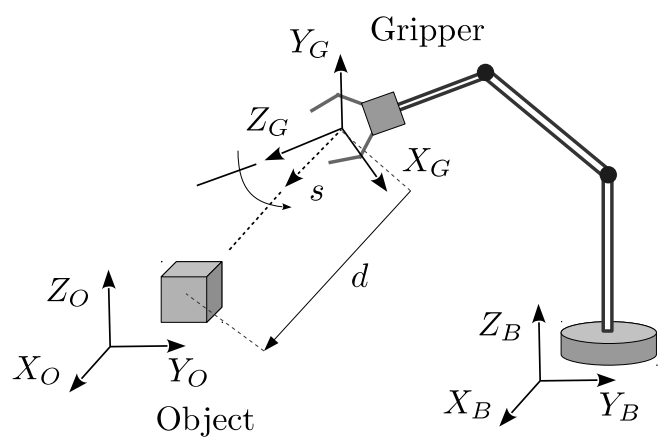

Fig. 1. An illustration of the manipulator with the gripper and the object to be grasped, along with explanatory notations and the attached frames of reference.

For the purposes of this work, we assume the presence of an exteroceptive sensor (e.g., an external camera) able to retrieve the $3 \mathrm{D}$ pose of $\mathcal{F}_{O}$ w.r.t. $\mathcal{F}_{B}$, represented by $\left({ }^{B} \boldsymbol{P}_{O},{ }^{B} \boldsymbol{R}_{O}\right) \in \mathbb{R}^{3} \times S O(3)$ (see also [3]). On the other hand, the $3 \mathrm{D}$ pose of $\mathcal{F}_{G}$ w.r.t. $\mathcal{F}_{B}$, namely $\left({ }^{B} \boldsymbol{P}_{G},{ }^{B} \boldsymbol{R}_{G}\right) \in$ $\mathbb{R}^{3} \times S O(3)$, can be retrieved through forward kinematics.

Following this notation, and considering the position of the object to be fixed in the scene, the relative gripper/object pose in the gripper frame $\mathcal{F}_{G}$ can be obtained as ${ }^{G} \boldsymbol{R}_{O}=$ ${ }^{G} \boldsymbol{R}_{B}{ }^{B} \boldsymbol{R}_{O}$ and ${ }^{G} \boldsymbol{P}_{O}={ }^{G} \boldsymbol{R}_{B}\left({ }^{B} \boldsymbol{P}_{O}-{ }^{B} \boldsymbol{P}_{G}\right)$. Finally, we consider $\left(\boldsymbol{v}_{G}, \boldsymbol{\omega}_{G}\right) \in \mathbb{R}^{6}$, the linear and angular velocity of the gripper expressed in its own frame, to be the control inputs for the slave manipulator arm.

\section{B. Slave side}

The proposed shared-control architecture aims at facilitating the pre-grasp approaching phase of the human operator, by enabling an autonomous algorithm to control a convenient subset $s \in \mathbb{R}^{m}, m<6$ of the gripper/object relative pose as a "primary" task. The operator is then able to control the robot in the remaining null space directions. At the same time, the haptic interface provides force cues to avoid encountering potential constraints of the robotic system (e.g., joint limits).

A convenient definition of the primary task for the pregrasp approach phase is to automatically orient the gripper towards the object. To this end, our control variable $s$ is chosen to be

$$
\boldsymbol{s}=\frac{{ }^{G} \boldsymbol{P}_{O}}{\left\|\boldsymbol{P}_{O}\right\|} \in \mathbb{S}^{2},
$$

as shown in Fig. 1. Since $Z_{G}$ should be aligned with the gripper approaching direction, the desired value of $s$ would 
then be $s_{d}=Z_{G}$, and the resulting control law associated with the primary task is described by

$$
\left[\begin{array}{c}
\boldsymbol{v}_{G} \\
\boldsymbol{\omega}_{G}
\end{array}\right]=k_{G} \boldsymbol{L}_{s}^{\dagger}\left(\boldsymbol{s}_{d}-\boldsymbol{s}\right), \quad k_{G}>0,
$$

where $k_{G}$ is a control gain, and $\boldsymbol{L}_{s}^{\dagger}$ represents the MoorePenrose pseudo-inverse of the interaction matrix $\boldsymbol{L}_{s}$ associated with $s$ and defined by

$$
\boldsymbol{L}_{s}=\left[\begin{array}{ll}
-\frac{1}{d} \boldsymbol{P}_{s} & {[\boldsymbol{s}]_{\times}}
\end{array}\right] \in \mathbb{R}^{3 \times 6},
$$

where $\boldsymbol{P}_{s}=\boldsymbol{I}-\boldsymbol{s s ^ { T }}$ is the orthogonal projector onto the tangent space of the unit sphere $\mathbb{S}^{2}$ at $s, d=\left\|{ }^{G} \boldsymbol{P}_{O}\right\|$, and $[\cdot]_{\times}$is the skew-symmetric matrix operator, see also [3]. Note that since $s$ is the unit norm defined in (1), the rank of $\boldsymbol{L}_{s} \in \mathbb{R}^{3 \times 6}$ is limited to 2 .

Let $\boldsymbol{N}_{B}=\left[\ldots \boldsymbol{n}_{i} \ldots\right] \in \mathbb{R}^{6 \times 4}$ be a basis spanning the null space of $\boldsymbol{L}_{s}$. The human operator is given command over these 4 null-space motion directions, and the resulting control law governing the full motion of the gripper is

$$
\left[\begin{array}{c}
\boldsymbol{v}_{G} \\
\boldsymbol{\omega}_{G}
\end{array}\right]=k_{G} \boldsymbol{L}_{s}^{\dagger}\left(\boldsymbol{s}_{d}-\boldsymbol{s}\right)+\sum_{i=1}^{n} \lambda_{i} \boldsymbol{n}_{i}, \quad k_{G}>0,
$$

where $\boldsymbol{\lambda}=\left[\ldots \lambda_{i} \ldots\right] \in \mathbb{R}^{4}$ indicates the pseudo-velocity commands of the human operator.

As described in [3], the null-space basis $\boldsymbol{N}_{B}$ is chosen to be

$$
\boldsymbol{n}_{1}=\left[\begin{array}{l}
\boldsymbol{s} \\
\mathbf{0}
\end{array}\right], \boldsymbol{n}_{2}=\left[\begin{array}{l}
\mathbf{0} \\
\boldsymbol{s}
\end{array}\right], \boldsymbol{n}_{3}=\left[\begin{array}{c}
-[\boldsymbol{s}]_{\times} \boldsymbol{e}_{y} \\
-\boldsymbol{P}_{s} \boldsymbol{e}_{y} / d
\end{array}\right], \boldsymbol{n}_{4}=\left[\begin{array}{c}
{[\boldsymbol{s}]_{\times} \boldsymbol{e}_{x}} \\
\boldsymbol{P}_{s} \boldsymbol{e}_{x} / d
\end{array}\right],
$$

with $\boldsymbol{e}_{x}=\left[\begin{array}{lll}1 & 0 & 0\end{array}\right]^{T}$ and $\boldsymbol{e}_{y}=\left[\begin{array}{lll}0 & 1 & 0\end{array}\right]^{T}$. This provides the human operator with a clear and decoupled physical interpretation of the directions she/he is commanding. A visual illustration of the four null-space motion directions is illustrated in Fig. 2.

\section{Master side}

The human operator uses a grounded haptic interface to command the slave manipulator (i.e., the four aforementioned null-space motion directions). The haptic interface has $6 \mathrm{DoF}$, two of which are blocked when the shared control algorithm is actuated. Figure 2 shows the degrees of freedom the operator can command.

The master device is modeled as a generic, gravity precompensated, mechanical system,

$$
\boldsymbol{M}\left(\boldsymbol{x}_{M}\right) \ddot{\boldsymbol{x}}_{M}+\boldsymbol{C}\left(\boldsymbol{x}_{M}, \dot{\boldsymbol{x}}_{M}\right) \dot{\boldsymbol{x}}_{M}=\boldsymbol{\tau}+\boldsymbol{\tau}_{h},
$$

where $\boldsymbol{x}_{M} \in \mathbb{R}^{4}$ represents the device configuration vector, $\boldsymbol{M}\left(\boldsymbol{x}_{M}\right) \in \mathbb{R}^{4 \times 4}$ is the positive-definite and symmetric inertia matrix, $\boldsymbol{C}\left(\boldsymbol{x}_{M}, \dot{\boldsymbol{x}}_{M}\right) \in \mathbb{R}^{4 \times 4}$ consists of the Coriolis/centrifugal terms, and $\tau, \tau_{h} \in \mathbb{R}^{4}$ are the control and human forces, respectively.

Differently from [3], we considered here two control modes:

- position-velocity: positions of the master handle are used to command the velocities of the slave robot (this is the same modality also employed in [3]);


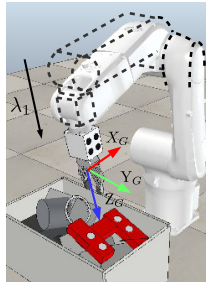

(b)



(c)



(d)

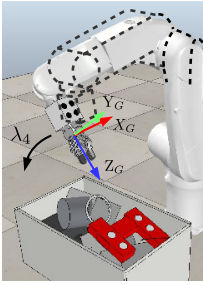

(e)
Fig. 2. A visualization of the four null-space directions defined in (5). (a): the four directions and the associated pseudo-velocity commands $\lambda_{i}$ projected on the slave and the master. (b-e): an illustration of the motion resulting from actuating each individual direction $\boldsymbol{n}_{i}$.

- velocity-velocity: velocities of the master handle are coupled to the the velocities of the slave robot, modulo a constant roto-translation scaling factor.

The details of both modes, along with the corresponding force cues, are illustrated below.

1) Position-velocity control: In the position-velocity control mode, the master/slave coupling is governed by

$$
\boldsymbol{\lambda}=\boldsymbol{K}_{\lambda} \boldsymbol{x}_{M},
$$

where $\boldsymbol{K}_{\lambda} \in \mathbb{R}^{4 \times 4}$ is a diagonal matrix of positive scaling factors. The operator then needs to adjust the position of the master device to control the speed along the null-space directions $n_{i}$.

In this modality, force cues are designed with two goals in mind. First, we want to guide the user away from constraints in the robotic system (e.g., joint limits), and, second, we want the operator to easily find the "zero-velocity" position on the master side, i.e., $\boldsymbol{x}_{M}=\mathbf{0}$, for possibly sending a zero velocity command to the slave arm. Toward this objective, we define the feedback action as

$$
\boldsymbol{\tau}=-\boldsymbol{B}_{M} \dot{\boldsymbol{x}}_{M}-\boldsymbol{K}_{M} \boldsymbol{x}_{M}+\boldsymbol{f} .
$$

where $\boldsymbol{K}_{M} \in \mathbb{R}^{4 \times 4}$ and $\boldsymbol{B}_{M} \in \mathbb{R}^{4 \times 4}$ are positive definite matrices, indicating the stiffness and damping factors of a spring pushing the master handle toward $\boldsymbol{x}_{M}=0$. In this way, whenever the user does not apply a force on the master handle, the latter moves back to $\boldsymbol{x}_{M}=\mathbf{0}$, causing the slave robot to stop (see (7)). The damping $\boldsymbol{B}_{M}$ is chosen so as to keep the teleoperation system stable. 
The term $\boldsymbol{f}=\left[\ldots f_{i} \ldots\right]^{T} \in \mathbb{R}^{4}$ accounts for the force cues providing the user with information on the feasibility of her/his commands against possible constraints. Of course, the constraints will vary depending on the application, and they can be modeled as a positive cost function quantifying the proximity of the system to the corresponding constraint. Let $H(\boldsymbol{q}) \geq 0$ be a cost function where $\boldsymbol{q} \in \mathbb{R}^{6}$ is the joint configuration vector of the slave manipulator. Each force cue $f_{i}$ aims at informing the user of the impact of actuating the corresponding motion direction $\boldsymbol{n}_{i}$ on the cost function $H(\boldsymbol{q})$, i.e., $(i)$ the direction (positive or negative) which would increase the cost function and drive the system closer to the constraint, and (ii) the magnitude, or the rate, by which moving along that direction would increase $H(\boldsymbol{q})$. This is achieved by setting

$$
f_{i}=-\left(\frac{\partial H(\boldsymbol{q})}{\partial \boldsymbol{q}}\right)^{T} \boldsymbol{J}_{G}^{-1}(\boldsymbol{q}) \boldsymbol{n}_{i}
$$

i.e., by projecting the joint motion caused by the $i$-th nullspace direction $\boldsymbol{n}_{i}$ onto the negative gradient of $H(\boldsymbol{q})$. Matrix $\boldsymbol{J}_{G}(\boldsymbol{q}) \in \mathbb{R}^{6 \times 6}$ is the geometric Jacobian mapping the joint velocities $\dot{\boldsymbol{q}}$ onto the gripper linear/angular velocities $\left(\boldsymbol{v}_{G}, \boldsymbol{\omega}_{G}\right)$.

In our experiments, proximity to joint limits was considered as a representative constraint of the slave system, and the cost function $H(\boldsymbol{q})$ was designed as a quadratic penalty cost function:

$$
H(\boldsymbol{q})=k_{H} \sum_{i=1}^{6} h_{i}(\boldsymbol{q})
$$

with

$$
h_{i}(\boldsymbol{q})=\left\{\begin{array}{ll}
\left(q_{i}-\left(q_{i, \max }-q_{t h}\right)\right)^{2}, & \text { if } q_{i} \geq q_{i, \max }-q_{t h} \\
\left(q_{i, \min }+q_{t h}-q_{i}\right)^{2}, & \text { if } q_{i} \leq q_{i, \min }+q_{t h}, \\
0, & \text { otherwise }
\end{array},\right.
$$

where $\left(q_{i, \max }, q_{i, \min }\right)$ are the maximum/minimum range for the $i$-th joint, $q_{t h}$ is a user-defined threshold defining the activation region inside which the user will receive a force feedback, and $k_{H}$ is a scaling factor.

The main advantage of this position-velocity control mode is that the operator is not limited by the workspace of the master device, making it quite convenient when using a master interface with a small workspace w.r.t. the slave robot. However, mapping positions to velocities may be less intuitive than simply mapping velocities to velocities. For this reason, this shared control modality may increase the cognitive load on the operator performing the task, at least during the initial training phases.

2) Velocity-velocity control: In the velocity-velocity control mode, the master/slave coupling is governed by

$$
\boldsymbol{\lambda}=\boldsymbol{K}_{\lambda, v e l}\left[\begin{array}{c}
\boldsymbol{v}_{M} \\
\boldsymbol{\omega}_{M}
\end{array}\right],
$$

where, as before, $\boldsymbol{K}_{\lambda, v e l} \in \mathbb{R}^{4 \times 6}$ is a diagonal matrix of positive scaling factors, while $\left(\boldsymbol{v}_{M}, \boldsymbol{\omega}_{M}\right)$ is the linear/angular velocity of the master device. A button on the master handle works as a clutch. Clutching allows the user to pause the remote operation, move the haptic device to a more comfortable or suitable position, and then resume the control of the robot. This approach is commonly used to address issues of limited workspace on the master side.

Force cues are then defined simply as

$$
\boldsymbol{\tau}=-\boldsymbol{B}_{M} \dot{\boldsymbol{x}}_{M}+\boldsymbol{f}
$$

with $f$ having the same definition as (9).

The clear advantage of this velocity-velocity control modality is the lower cognitive load required with respect to the position-velocity mode of Sec. II-C.1. In the velocityvelocity case, in fact, the user's commands are directly reflected to the slave (e.g., the robot stops when the operator stops, and it moves when the operator moves). On the other hand, in position-velocity mode, this relationship is not as evident for the operator, who needs to think a bit more about the impact of his actions on the slave manipulator. However, limitations in the workspace of the master device are more evident in velocity-velocity mode than in position-velocity mode, with the former requiring clutching. The amount of clutching required is directly dependent on the scaling factor between the master/slave workspaces. If the scaling is high, the user will not be required to clutch a lot. However, this may results in fast and abrupt movements of the slave robot. On the other hand, if the scaling is low, the user will be able to more precisely and smoothly control the motion of the slave robot. However, more clutching will be necessary.

\section{EXPERIMENTAL EVALUATION}

In order to demonstrate the feasibility and effectiveness of our method, we conducted a telemanipulation experiment in a real environment. We compared the proposed shared-control approach with a more classic teleoperation architecture, in which the human operator is in charge of controlling all the DoF of the slave manipulator. Moreover, we also compared two different approaches to control the motion of the robotic manipulator through the haptic interface (position-velocity vs. velocity-velocity).

\section{A. Experimental setup}

Figure 3 shows the experimental setup. The master system is composed of the Haption Virtuose 6D haptic device (Haption, France), a high performance force feedback device with three active translational DoF and three active rotational DoF. The maximum force/torque is about $30 \mathrm{~N} / 3 \mathrm{Nm}$, the workspace has a spherical shape with an approximated radius of $0.9 \mathrm{~m}$, and the device exchanges data over Ethernet with a computer at $1 \mathrm{kHz}$. The slave system is composed of a 6 -DoF Viper S850 robotic arm carrying a pneumatic parallel gripper. A wooden object with dimensions $21 \times 9 \times 3 \mathrm{~cm}$ and weight $280 \mathrm{~g}$ is placed on a table in front of the robotic manipulator. The user had a direct view of the slave system and the object to be grasped. 


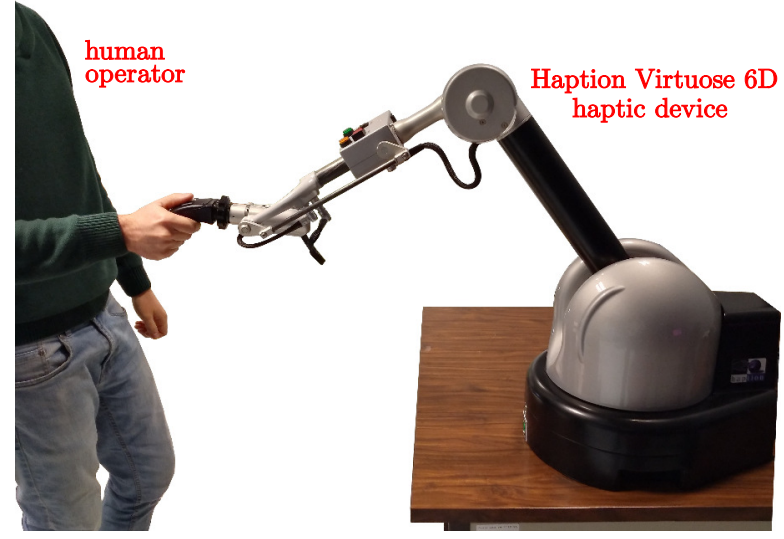

(a) Master side

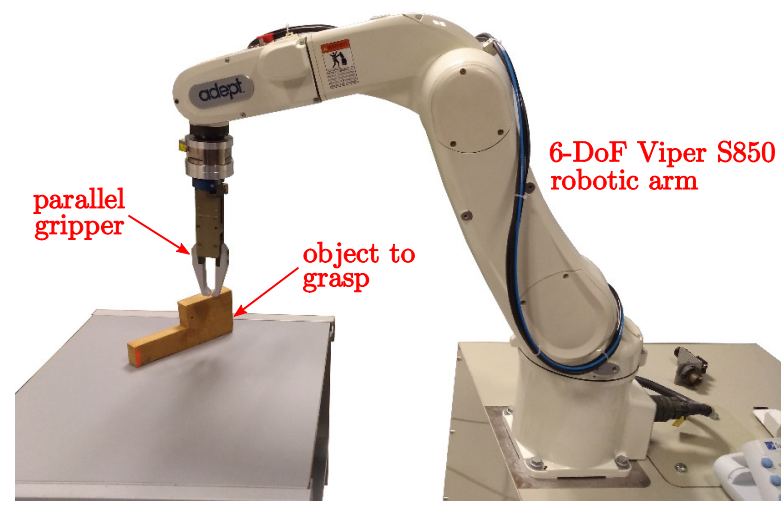

(b) Slave side

Fig. 3. Experimental setup. The master system is composed of the Haption Virtuose 6D haptic device, while the the slave system is composed of a 6 -DoF Viper S850 robotic arm carrying a pneumatic parallel gripper. A wooden object with dimensions $21 \times 9 \times 3 \mathrm{~cm}$ and weight $300 \mathrm{~g}$ is placed on a table in front of the robotic manipulator.

\section{B. Experimental conditions and task}

Participants were required to control the motion of the robotic manipulator and gripper to grasp the wooden piece and lift it from the ground. The task started when the manipulator moved for the very first time and it was considered successfully completed when the object was lifted from the ground.

We considered two different ways of commanding the motion of the robot through the haptic interface (positionvelocity vs. velocity-velocity, see Sec. II-C) and two different levels of human involvement in the control (shared control vs. teleoperation), ending up with four different experimental conditions:

S+PV: shared-control with position-velocity motion control, where the subject controls only 4 DoF of the manipulator, and positions of the haptic device are mapped into velocities of the manipulator (see Sec. II-C.1);

S+VV: shared-control with velocity-velocity motion control, where the subject controls only 4 DoF of the manipulator, and velocities of the haptic device are mapped into velocities of the manipulator (see
Sec. II-C.2)

$\mathrm{T}+\mathrm{PV}$ : teleoperation with position-velocity motion control, where the subject controls all the 6 DoF of the manipulator, and positions of the haptic device are mapped into velocities of the manipulator;

$\mathrm{T}+\mathrm{VV}$ : teleoperation with velocity-velocity motion control, where the subject controls all the $6 \mathrm{DoF}$ of the manipulator, and velocities of the haptic device are mapped into velocities of the manipulator.

The shared-control architecture, employed in conditions $\mathrm{S}+\mathrm{PV}$ and $\mathrm{S}+\mathrm{VV}$, is detailed in Sec. II.

In $\mathrm{T}+\mathrm{PV}$ and $\mathrm{T}+\mathrm{VV}$ conditions, the subject is in full control of the manipulator's 6 DoF. In this respect, the master/slave coupling in $\mathrm{T}+\mathrm{PV}$ is

$$
\left[\begin{array}{c}
\boldsymbol{v}_{G} \\
\boldsymbol{\omega}_{G}
\end{array}\right]=\boldsymbol{K}_{P V} \boldsymbol{x}_{M},
$$

where the configuration vector of the master device, $\boldsymbol{x}_{M}$, is now in $\mathbb{R}^{6}$ and $\boldsymbol{K}_{P V} \in \mathbb{R}^{6 \times 6}$ is a matrix mapping $\boldsymbol{x}_{M}$ to velocity commands on the slave side. Similarly to Sec. II-C.1, the force cues fed to the user are

$$
\boldsymbol{\tau}=-\boldsymbol{B}_{M} \dot{\boldsymbol{x}}_{M}-\boldsymbol{K}_{M} \boldsymbol{x}_{M}+\boldsymbol{f},
$$

where $\boldsymbol{B}_{M} \in \mathbb{R}^{6 \times 6}$ and $\boldsymbol{K}_{M} \in \mathbb{R}^{6 \times 6}$ are the damping and stiffness matrices of a spring pushing the master handle back to the "zero velocity" position, and, from (9), $\boldsymbol{f}$ is defined as

$$
\boldsymbol{f}=-\left(\frac{\partial H(\boldsymbol{q})}{\partial \boldsymbol{q}}\right)^{T} \boldsymbol{J}_{G}^{-1}(\boldsymbol{q})
$$

since now no primary task is present and, therefore, the null-space basis $\boldsymbol{N}_{B}$ is just the identity matrix.

On the other hand, in the T+VV condition the coupling is

$$
\left[\begin{array}{c}
\boldsymbol{v}_{G} \\
\boldsymbol{\omega}_{G}
\end{array}\right]=\boldsymbol{K}_{V V}\left[\begin{array}{c}
\boldsymbol{v}_{M} \\
\boldsymbol{\omega}_{M}
\end{array}\right]
$$

where $\boldsymbol{K}_{V V} \in \mathbb{R}^{6 \times 6}$ is a diagonal scaling matrix. Haptic feedback is then designed similarly to Sec. II-C.2, with

$$
\boldsymbol{\tau}=-\boldsymbol{B}_{M} \dot{\boldsymbol{x}}_{M}+\boldsymbol{f}
$$

where $\boldsymbol{f}$ follows (14).

\section{Participants}

Ten right-handed subjects (average age 27.2) participated in the study. Three of them had previous experience with haptic interfaces. None of the participants reported any deficiencies in their visual or haptic perception abilities. The experimenter explained the procedures and spent about two minutes adjusting the setup to be comfortable before the subject began the experiment. Each subject then spent about three minutes practicing the control of the telemanipulation system before starting the experiment. Each subject carried out 8 randomized repetitions of the grasping task, 2 for each experimental condition. A video showing trials in all experimental conditions is available as supplemental material. 


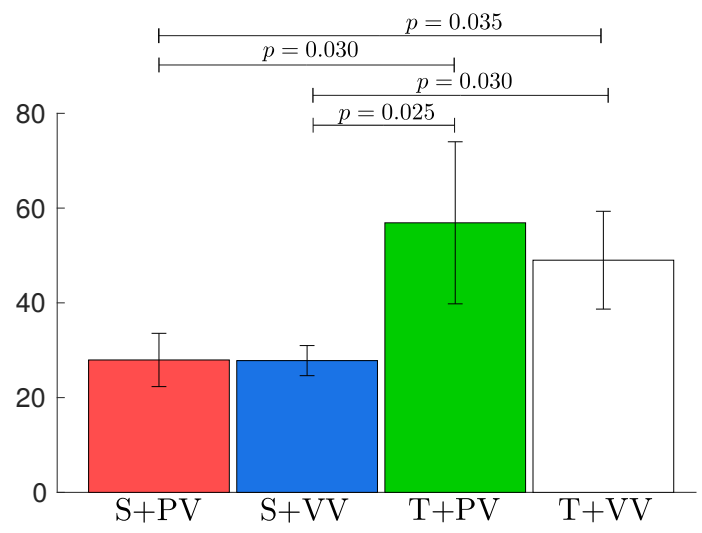

(a) Completion time (s)

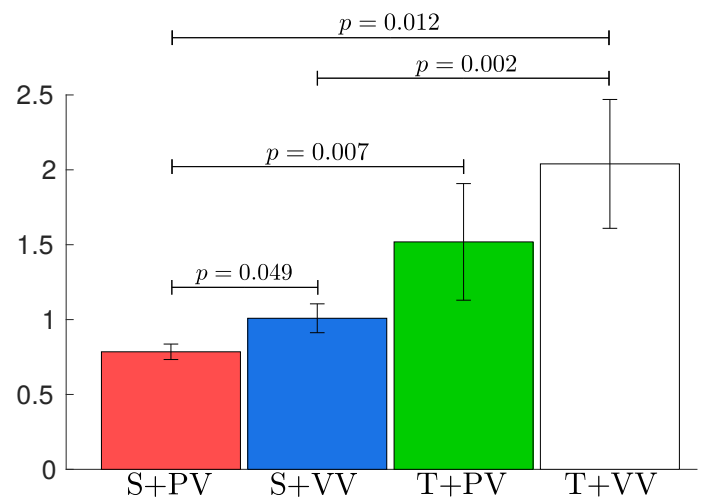

(b) Trajectory length (m)

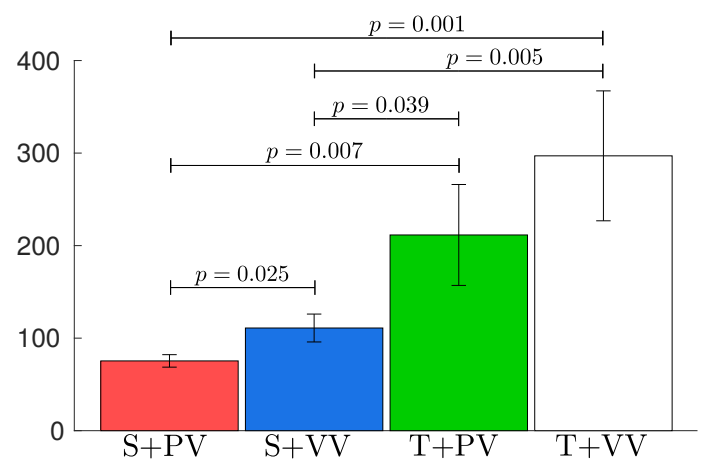

(c) Angular motion (deg)

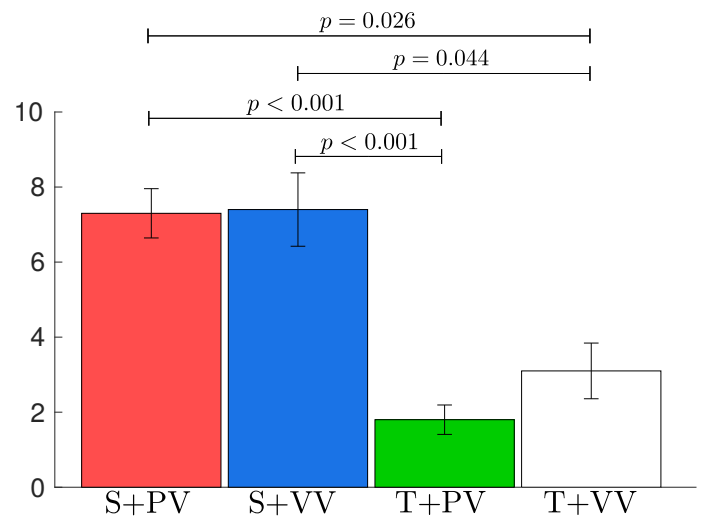

(d) Perceived effectivess

Fig. 4. Experimental evaluation. Mean and 95\% confidence interval of (a) completion time, (b) trajectory length, (c) angular motion, and (d) perceived effectiveness of the four feedback conditions are plotted.

\section{Results}

To evaluate the effectiveness of our system in grasping the considered object, the usefulness of the proposed sharedcontrol approach, and the effectiveness of haptic stimuli to render robotic setup constraints, we recorded $(i)$ the completion time, $(i i)$ the linear trajectory followed by the robotic end-effector, (iii) the angular motion of the robotic end-effector, and $(i v)$ the perceived effectiveness of the different conditions. To compare the different metrics, we ran both two-way and one-way repeated-measures ANOVA tests on the data. In the two-way analysis, motion control (positionvelocity vs. velocity-velocity) and human involvement in the control (shared control vs. teleoperation) were treated as within-subject factors. All data passed the Shapiro-Wilk normality test. Sphericity was assumed for variables with only two levels of repeated measures. The two-way analysis enables us to understand the role of each variable considered within-subject factor, while the one-way analysis provides us with an overview on the performances of the four conditions.

Figure 4a shows the average task completion time. The twoway ANOVA test revealed a statistically significant change in the task completion time for the human involvement in the control variable (shared control vs. teleoperation, $\mathrm{F}(1,9)$ $=25.852, p=0.001)$. The interaction effect between these two factors was not statistically significant. The one-way ANOVA test revealed a statistically significant change in the task completion time across the conditions $(\mathrm{F}(3,27)=9.312$, $p<0.001$ ). Post hoc analysis with Bonferroni adjustments revealed a statistically significant difference between $\mathrm{S}+\mathrm{VV}$ vs. $\mathrm{T}+\mathrm{VV}(p=0.030), \mathrm{S}+\mathrm{VV}$ vs. $\mathrm{T}+\mathrm{PV}(p=0.035), \mathrm{S}+\mathrm{PV}$ vs. $\mathrm{T}+\mathrm{VV}(p=0.031)$, and $\mathrm{S}+\mathrm{PV}$ vs. $\mathrm{T}+\mathrm{PV}(p=0.025)$. The Bonferroni correction is used to reduce the chances of obtaining false-positive results when multiple pair-wise tests are performed on a single set of data.

Figure $4 \mathrm{~b}$ shows the average linear motion covered by the robotic gripper during the task. The two-way ANOVA test revealed a statistically significant change in the trajectory length for both the human involvement in the control $(\mathrm{F}(1$, $9)=30.968, p<0.001)$ and the motion control type (velocity vs. position, $\mathrm{F}(1,9)=9.035, p=0.015$ ) variables. The interaction effect between these two factors was not statistically significant. The one-way ANOVA test revealed a statistically significant change in the trajectory length across the conditions $(\mathrm{F}(1.929,17.360)=14.072, p<0.001)$. Post hoc analysis with Bonferroni adjustments revealed a statistically significant difference between $\mathrm{S}+\mathrm{VV}$ vs. S+PV $(p=0.049), \mathrm{S}+\mathrm{VV}$ vs. $\mathrm{T}+\mathrm{VV}(p=0.043), \mathrm{S}+\mathrm{VV}$ vs. $\mathrm{T}+\mathrm{PV}$ $(p=0.002)$, and $\mathrm{S}+\mathrm{PV}$ vs. $\mathrm{T}+\mathrm{PV}(p=0.012)$.

Figure $4 \mathrm{c}$ shows the average angular motion covered by the robotic gripper during the task. The two-way ANOVA test revealed a statistically significant change in the angular motion for both the human involvement in the control $(\mathrm{F}(1,9)$ $=39.350, p<0.001$ ) and the motion control type (positionvelocity vs. velocity-velocity, $\mathrm{F}(1,9)=8.202, p=0.015$ ) variables. The interaction effect between these two factors was not statistically significant. The one-way ANOVA test 
revealed a statistically significant change in the trajectory length across the conditions $(\mathrm{F}(3,27)=12.994, p<0.001)$. Post hoc analysis with Bonferroni adjustments revealed a statistically significant difference between $\mathrm{S}+\mathrm{VV}$ vs. S+PV $(p=0.025), \mathrm{S}+\mathrm{VV}$ vs. $\mathrm{T}+\mathrm{VV}(p=0.007), \mathrm{S}+\mathrm{VV}$ vs. $\mathrm{T}+\mathrm{PV}$ $(p=0.001), \mathrm{S}+\mathrm{PV}$ vs. $\mathrm{T}+\mathrm{VV}(p=0.039)$, and $\mathrm{S}+\mathrm{PV}$ vs. $\mathrm{T}+\mathrm{PV}(p=0.005)$.

Immediately after the experiment, subjects were also asked to report the effectiveness of each feedback condition in completing the given task using bipolar Likert-type ninepoint scales. Fig. 4d shows the perceived effectiveness for the four experimental conditions. A Friedman test showed a statistically significant difference between the means of the four feedback conditions $\left(\chi^{2}(3)=26.753, p<0.001, a=\right.$ $0.05)$. The Friedman test is the non-parametric equivalent of the more popular repeated-measures ANOVA. The latter is not appropriate here since the dependent variable was measured at the ordinal level. Post hoc analysis with Bonferroni adjustments revealed a statistically significant difference between $\mathrm{S}+\mathrm{VV}$ vs. $\mathrm{T}+\mathrm{VV}(p<0.001), \mathrm{S}+\mathrm{VV}$ vs. $\mathrm{T}+\mathrm{PV}$ $(p=0.026), \mathrm{S}+\mathrm{PV}$ vs. $\mathrm{T}+\mathrm{VV}(p<0.001)$, and $\mathrm{S}+\mathrm{PV}$ vs. $\mathrm{T}+\mathrm{PV}(p=0.044)$.

Finally, all ten subjects found conditions using the sharedcontrol approach to be the most effective at completing the grasping task. Seven subjects out of ten chose the sharedcontrol condition employing velocity-velocity control to be the most effective.

\section{DISCUSSION, CONCLUSIONS, AND FUTURE WORK}

This paper presents the evaluation of a generic sharedcontrol architecture for robotic telemanipulation, whose objective is to help a human operator in completing a predefined grasping task. While in the literature we can find several works presenting different shared-control architecture, very few evaluate their task performance and user's acceptance in principled human subjects experiments. In our work, we tested the proposed shared-control architecture in a real environment composed a 6-DoF robotic manipulator, equipped with a parallel gripper, whose motion is controlled through a 6DoF grounded haptic interface. The autonomous algorithm is in charge of controlling $2 \mathrm{DoF}$ of the robotic manipulator, keeping the gripper always oriented toward the object to grasp. The gripper is therefore constrained to move on the surface of a virtual sphere centered on the object. The human operator is able to move the gripper across the surface of the sphere and toward/away from the object (i.e., changing the radius of the sphere).

In order to demonstrate the feasibility and effectiveness of our method, we conducted a remote telemanipulation experiment where ten human subjects were asked to control the motion of the 6-DoF telemanipulator to grasp a wooden object. We tested the performance of the proposed sharedcontrol system against a more classic teleoperation approach, in which the human user was able to freely control all the degrees of freedom of the robotic manipulator. Moreover, we considered two ways of controlling the motion of the robot through the haptic interface - velocity-velocity and positionvelocity, - ending up with four experimental conditions. As a measure of performance, we considered the average completion time, trajectory length, angular motion, and perceived effectiveness.

We carried out a 2-way statistical analysis to be able to separately compare the different ways of commanding the motion of the robot through the haptic interface (velocityvelocity vs. position-velocity) and the different levels of human involvement in the control (shared-control vs. teleoperation). After that, we also carried out a 1-way statistical analysis to be able to compare all the four composite experimental conditions at the same time. Results showed that, in all the considered metrics, the shared-control approach significantly outperformed the more classic teleoperation architecture. Moreover, all the subjects preferred the sharedcontrol architecture with respect to teleoperation. This proves our hypothesis that shared-control can be a viable and very effective approach to improve currently-available teleoperation systems in remote manipulation tasks. However, it is important to notice that our subjects were not expert in using the experimental setup. In this respect, it may happen that the recorded significant difference in performance between shared control vs. teleoperation might become less significant in the presence of experienced users. This is something we plan to extensively study in the coming future, since it is particularly relevant for the context of our target project, RoMaNS, in which the operators in charge of sorting and segregating the nuclear waste can be skilled and experienced.

In addition to this first result, the 1-way statistical analysis gave us insights about the differences between the composite conditions. Results show a significant difference between $\mathrm{S}+\mathrm{PV}$ vs. $\mathrm{S}+\mathrm{VV}$ in the trajectory length and angular motion metrics, with the former condition outperforming the latter. This result came as a surprise, since it is in contrast with the results of the user experience evaluation. In fact, both the perceived effectiveness and the choice of the preferred condition clearly show that users preferred conditions employing velocity-velocity control with respect to position-velocity control. In this respect, all subjects complained that positionvelocity conditions required more attention and, in general, a higher cognitive load. Three subjects, who indicated S+VV as their preferred condition, asserted that they would have probably preferred condition $\mathrm{S}+\mathrm{PV}$ if the task would have required more time to complete, since $\mathrm{S}+\mathrm{PV}$ does not require clutching (see Sec. II-C).

Clutching is indeed another interesting point to discuss. Even in the velocity-velocity control approach, given a grounded haptic interface and a grounded slave manipulator, it is always possible to define an appropriate scaling factor between master and slave velocities such that the operator does not require clutching. However, as the difference between the master and slave workspaces increases, this mapping requires higher and higher gains, resulting in a telemanipulation system very hard to control, since the operator's accuracy/resolution in positioning the slave arm is 
degraded. The RoMaNS project presents us with the perfect example: the custom rig at the National Nuclear Laboratory is composed of (i) the same grounded haptic interface we are using in this paper, and (ii) a 500-kg-payload Kuka KR 500 manipulator. Although it is theoretically possible to map the workspace of the Virtuose 6D to the (much) larger workspace of the KUKA robot, this would result in very high motion gains (i.e., a small movement of the master interface would cause a big movement of the slave robot). For this reason, we decided to implement the velocity-velocity modality using the clutch. In this respect, we are also interested in understanding how to best tune the master-slave motion scaling factor, with the final objective of finding a good trade-off between high precision of movement and low need of clutching.

It is important to highlight that the proposed shared-control architecture is independent from the distribution of the DoF between the autonomous controller and the human operator. While having the autonomous controller keeping the gripper oriented toward the object seemed a natural choice for our task, the underlying shared-control architecture is agnostic to the task, the primary task variables, and the level of human involvement. In this respect, we are planning to study how the number of DoF controlled by the autonomous controller affects the task performance. For example, a system could use a highly-autonomous shared-control approach (i.e., many DoF managed by the autonomous controlled) when it is operated by novices, while it could implement a lowly-autonomous sharedcontrol approach (i.e., few DoF managed by the autonomous controlled) when it is operated by experts. This flexible approach could be also useful when teaching new operators.

Although our approach has shown promising result, it significantly limits the control capabilities of the human users, who are not able to freely move the robot wherever they prefer. This could be a serious problem if something unexpected happens in the environment. In order to address this point, we are studying new ways of providing guidance information to the operators using ungrounded tactile stimuli, with the objective of providing them with information about what the autonomous controller thinks they should do, but without reducing their capabilities to control the motion of the robot. This is particularly relevant in those scenarios where, for reasons of responsibility, safety, and public acceptance, it is beneficial to provide a human operator with full control of the motion of the robot, e.g., robot-assisted surgery. Another approach is to use a second robot holding a camera to track the position of the gripper and the object, adjusting the behavior of the autonomous controller if the object moves away from its original position.

Finally, all subjects appreciated the presence of haptic feedback to provide information about the manipulator's joint limits. This approach enabled them to always complete the task successfully, pushing them away from dangerous robot configurations in a very intuitive and non-obtrusive way. Subjects described the feeling due to the haptic feedback "as if the system was trying to nudge them towards a safer configuration of the robot."

\section{ACKNOWLEDGMENTS}

This work was supported by the EU H2020 RoMaNS project 645582 .

\section{REFERENCES}

[1] W.-K. Yoon, T. Goshozono, H. Kawabe, M. Kinami, Y. Tsumaki, M. Uchiyama, M. Oda, and T. Doi, "Model-based space robot teleoperation of ets-vii manipulator," IEEE Transactions on Robotics and Automation, vol. 20, no. 3, pp. 602-612, 2004.

[2] C. Freschi, V. Ferrari, F. Melfi, M. Ferrari, F. Mosca, and A. Cuschieri, "Technical review of the da vinci surgical telemanipulator," The International Journal of Medical Robotics and Computer Assisted Surgery, vol. 9, no. 4, pp. 396-406, 2013.

[3] F. Farraj, N. Pedemonte, and P. Robuffo Giordano, "A visual-based shared control architecture for remote telemanipulation," in IEEE/RSJ Int. Conf. on Intelligent Robots and Systems, 2016.

[4] A. M. G. Esfahani, F. Abi-Farraj, P. R. Giordano, and R. Stolkin, "Human-in-the-loop optimisation: mixed initiative grasping for optimally facilitating post-grasp manipulative actions," arXiv:1707.08147, 2017.

[5] N. Pedemonte, F. Farraj, and P. Robuffo Giordano, "Visual-based shared control for remote telemanipulation with integral haptic feedback," in IEEE Int. Conf. on Robotics and Automation, 2017.

[6] D. Aschenbrenner, M. Fritscher, F. Sittner, M. Krauß, and K. Schilling, Teleoperation of an Industrial Robot in an Active Production Line, 2015.

[7] J. Cecil, M. B. R. Kumar, Y. Lu, and V. Basallali, "A review of micro-devices assembly techniques and technology," The International Journal of Advanced Manufacturing Technology, vol. 83, no. 9-12, pp. 1569-1581, 2016.

[8] C. Pacchierotti, S. Scheggi, D. Prattichizzo, and S. Misra, "Haptic feedback for microrobotics applications: a review," Frontiers in Robotics and AI, vol. 3, no. 53, 2016.

[9] J. P. Trevelyan, S.-C. Kang, and W. R. Hamel, "Robotics in hazardous applications," in Springer Handbook of Robotics. Springer, 2008, pp. 1101-1126.

[10] S. Stramigioli, R. Mahony, and P. Corke, "A novel approach to haptic tele-operation of aerial robot vehicles," in Proc. IEEE Int. Conf. on Robotics and Automation, 2010, pp. 5302-5308.

[11] J. Jurmain, A. Blancero, J. Geiling, A. Bennett, C. Jones, J. Berkley, M. Vollenweider, M. Minsky, J. Bowersox, and J. Rosen, "Hazbot: Development of a telemanipulator robot with haptics for emergency response." American journal of disaster medicine, vol. 3, no. 2, pp. 87-97, 2007.

[12] R. R. Murphy, K. L. Dreger, S. Newsome, J. Rodocker, E. Steimle, T. Kimura, K. Makabe, F. Matsuno, S. Tadokoro, and K. Kon, "Use of remotely operated marine vehicles at minamisanriku and rikuzentakata japan for disaster recovery," in Proc. IEEE Int. Symp. on Safety, Security, and Rescue Robotics, 2011, pp. 19-25.

[13] M. Dittmar, "Nuclear energy: Status and future limitations," Energy, vol. 37, no. 1, pp. 35-40, 2012.

[14] N. E. Agency, Radioactive Waste in Perspective, 2010.

[15] N. Chopra, P. Berestesky, and M. W. Spong, "Bilateral teleoperation over unreliable communication networks," IEEE Transactions on Control Systems Technology, vol. 16, no. 2, pp. 304-313, 2008.

[16] E. Samur, Performance metrics for haptic interfaces. Springer Science \& Business Media, 2012.

[17] F. Abi-Farraj, T. Osa, N. Pedemonte, J. Peters, G. Neumann, and P. G. Robuffo, "A learning-based shared control architecture for interactive task execution," in IEEE Int. Conf. on Robotics and Automation, 2017.

[18] A. M. Okamura, "Methods for haptic feedback in teleoperated robotassisted surgery," Industrial Robot: An International Journal, vol. 31, no. 6, pp. 499-508, 2004.

[19] C. Pacchierotti, "Cutaneous haptic feedback in robotic teleoperation," 2015. 